\title{
Mexican mayflies : inventory and additions (Ephemeroptera)
}

\author{
R.P. Randolph ${ }^{1}$ \\ W.P. McCafferty 1
}

Keywords : Ephemeroptera, Mexico, distribution, new country records, new state records.

The 138 species and subspecies of Ephemeroptera now known to occur in Mexico, of which 35, approximately $25 \%$, are endemic, are listed along with distributional annotations. New Mexican records are included for Americabaetis lugoi Waltz \& McCafferty, Baetodes velmae Cohen \& Allen, Drunella coloradensis (Dodds), Farrodes otiesa Lugo-Ortiz \& McCafferty, Plauditus virilis (McDunnough), Tricorythodes fictus Traver, T. texanus Traver, and Varipes lasiobrachius Lugo-Ortiz \& McCafferty, the latter of which also constitutes a new continental record for the genus Varipes Lugo-Ortiz \& McCafferty in North America. Traverella sallei (Navás), n. comb., is transferred from the genus Thraulus.

\section{Ephéméroptères mexicains : inventaire et compléments (Ephemeroptera)}

Mots-clés : Ephemeroptera, Mexique, répartition, citations nouvelles pour le pays, citations nouvelles par états.

Les 138 espèces et sous-espèces d'éphéméroptères actuellement connues du Mexique, dont 35 - soit approximativement $25 \%$ - endémiques, sont listées avec des annotations sur leur répartition. Les citations suivantes sont nouvelles pour le Mexique : Americabaetis lugoi Waltz \& McCafferty, Baetodes velmae Cohen \& Allen, Drunella coloradensis (Dodds), Farrodes otiesa Lugo-Ortiz \& McCafferty, Plauditus virilis (McDunnough), Tricorythodes fictus Traver, T. texanus Traver, et Varipes lasiobrachius Lugo-Ortiz \& McCafferty. Cette dernière constitue aussi une nouvelle citation continentale pour le genre Varipes LugoOrtiz \& McCafferty en Amérique du Nord. Traverella sallei (Navás), n. comb., est transférée du genre Thraulus.

\section{Introduction}

Mexico is significant with respect to the biodiversity and biogeography of its flora and fauna. It has been recognized as having a high number of endemic species and distinct regional assemblages due, in part, to its widely varied topography, which results in highly localized regional climates (Anderson \& O'Brien 1996). Also, the region represents a transition zone between the Nearctic and Neotropical zones (Ramamoorthy et al. 1993, Anderson \& O'Brien 1996, McCafferty 1998). As a result, Mexico was ranked third worldwide with respect to its biodiversity by Mittermeier (1988). In light of this, we would expect the Mexican mayfly (Ephemeroptera) fauna also to be rich, but moderated to a certain extent by the dearth of perennial

1. Department of Entomology,.Purdue_University,_West-Lafayette, IN 47907, USA. stream habitats in the larger arid regions, mainly in northern Mexico.

The most recent comprehensive listing of the Mexican mayfly fauna was provided by McCafferty \& Lugo-Ortiz (1996), wherein 116 species were documented from the literature up to that time. By comparison, 143 species are known from the state of Indiana, USA (Randolph \& McCafferty 1998), a region with an area of $99,993 \mathrm{~km}^{2}$, and less than $5 \%$ the area of Mexico $\left(1,972,544 \mathrm{~km}^{2}\right)$. Nevertheless, of the 138 Mexican species and subspecies reviewed herein, 35 or approximately $25 \%$, are endemic.

Prior to the study by McCafferty \& Lugo-Ortiz (1996), Edmunds et al. (1976) had listed 63 nominal species from Mexico. Since then, works by Allen (1977, 1978, 1985), Allen \& Brusca (1978), Allen \& Cohen (1977), Allen \& Murvosh (1983, 1987abc), Cohen \& Allen (1978), Lugo-Ortiz \& McCafferty (1994ab, 1995ab, 1996abc), Waltz \& McCafferty (1987),_Lugo=Ortiz_et_al._(1994),,-and-McCafferty-\&Lugo-Ortiz (1998) have provided the majority of re- 
cent Mexican mayfly records. Since McCafferty \& Lugo-Ortiz (1996), studies by Domínguez et al. (1996), Wiersema (1998), Domínguez (1999), Wiersema \& Baumgardner (2000), Baumgardner \& McCafferty (2000), McCafferty \& Randolph (2000), and Randolph \& McCafferty (2000) have also contributed some additional Mexican data, including new synonyms. The biogeography of Mexican mayfly genera was addressed by McCafferty et al. (1992) and McCafferty (1998). McCafferty et al. (1997) provided an illustrated stage correlated identification key in Spanish to all known Mexican mayfly families. For a review of works dealing with Mexican mayflies prior to Edmunds et al. (1976), see Lugo-Ortiz \& McCafferty (1994a) and McCafferty \& Lugo-Ortiz (1996).

Herein we provide an up to date inventory of the Mexican mayfly species and subspecies, and provide new country and state records based on previously unstudied material. We list 138 species and subspecies among 11 families and 38 genera, and include eight new country records and 48 new state records.

Of the new country records, Varipes lasiobrachius Lugo-Ortiz \& McCafferty also represents a new continental record for the genus Varipes Lugo-Ortiz \& McCafferty. This distinctive species was recently described from Colombia and Ecuador by Lugo-Ortiz \& McCafferty (1998). Its presence in Mexico, and North America, is remarkable because only four other mayfly species [Euthyplocia hecuba (Hagen), Hexagenia albivitta (Walker), H. mexicana Eaton, and Leptohyphes nigripunctus Traver] are known to occur in both North and South America (McCafferty 1985, 1998).

With respect to other species reported here for Mexico for the first time, Americabaetis lugoi Waltz \& McCafferty and Farrodes otiesa Lugo-Ortiz \& McCafferty were previously known only from Costa Rica, respectively (Waltz \& McCafferty 1999, Lugo-Ortiz \& McCafferty 1996b), and Baetodes velmae Cohen \& Allen was known only from Panama (Cohen \& Allen 1978). Our discovery of Plauditus virilis (McDunnough) in Mexico also represents the first report of the genus Plauditus Lugo-Ortiz \& McCafferty south of the USA. Tricorythodes fictus Traver was previously known only from the southwestern USA (Traver 1935). Allen (1967) reported paratype material of $T$. edmundsi Allen from Tamaulipas. The recent examination by $\mathrm{N}$. A. Wiersema (pers. comm.) of that paratype material has shown that those specimens are actually misidentified T. texanus Traver. Drunella coloradensis (Dodds) was previously known throughout the western USA and Canada (Allen \& Edmunds 1962, McCafferty et al. 1997, McCafferty \& Randolph 1998).
We include the species Traverella sallei (Navás), $\mathrm{n}$. comb., in our inventory, although it had been inadvertently omitted previously by McCafferty \& Lugo-Ortiz (1996). This species was originally described in the genus Thraulus Eaton; however, Thraulus is now known to be limited to the Eastern Hemisphere (Peters and Edmunds 1970). Based on the genitalia figured by Navás (1935), this species is consistent with the genus Traverella Edmunds. Peter Grant (pers. comm.), who is currently revising Thraulus, has confirmed our assessment of the species. No specific location other than Mexico was provided by Navás, nor has the species been reported since its description.

The date ascribed to Homoeoneuria salviniae Eaton has sometimes been 1883 (e.g., Pescador and Peters 1980 , McCafferty 1996), but the species name appeared, and it was briefly described in 1881 (Eaton 1881). It should also be pointed out that the date ascribed to the species Camelobaetidius variabilis Wiersema is 1997. The date appearing on the issue of the journal in which the species was described (Wiersema 1998) was mailed and available in 1997, and thus the species name was available in 1997.

In the inventory below, known Mexican state distributions are provided for all species and full collection data are given for the new state and country records. Species are listed alphabetically by family, genus, and species. Mexican states are abbreviated as follows and are depicted in Figure 1: AG (Aguascalientes), BJ (Baja California), BS (Baja California Sur), $\mathrm{CH}$ (Chihuahua), CL (Coahuila), CM (Colima), CP (Campeche), CS (Chiapas), DF (Distrito Federal), DU (Durango), GJ (Guanajuato), GO (Guerrero), HD (Hidalgo), JA (Jalisco), MH (Michoacan), ML (Morelos), MX (state of Mexico), NL (Nuevo Leon), NT (Nayarit), OA (Oaxaca), PU (Puebla), QR (Quintana Roo), QT (Queretaro), SI (Sinaloa), SL (San Luis Potosi), SO (Sonora), TA (Tamaulipas), TB (Tabasco), TC (Tlaxcala), VC (Vera Cruz), YT (Yucatan), ZA (Zacatecas). Asterisks indicate species currently thought to be endemic to Mexico. For all species originally described from Mexico, states representing type localities for those species are also annotated. Except where otherwise indicated, all materials examined are deposited in the Purdue Entomological Research Collection, West Lafayette, Indiana.

\section{Baetidae}

2.1. Acentrella insignificans (McDunnough), 1926 $\mathrm{CH}$; SO. 


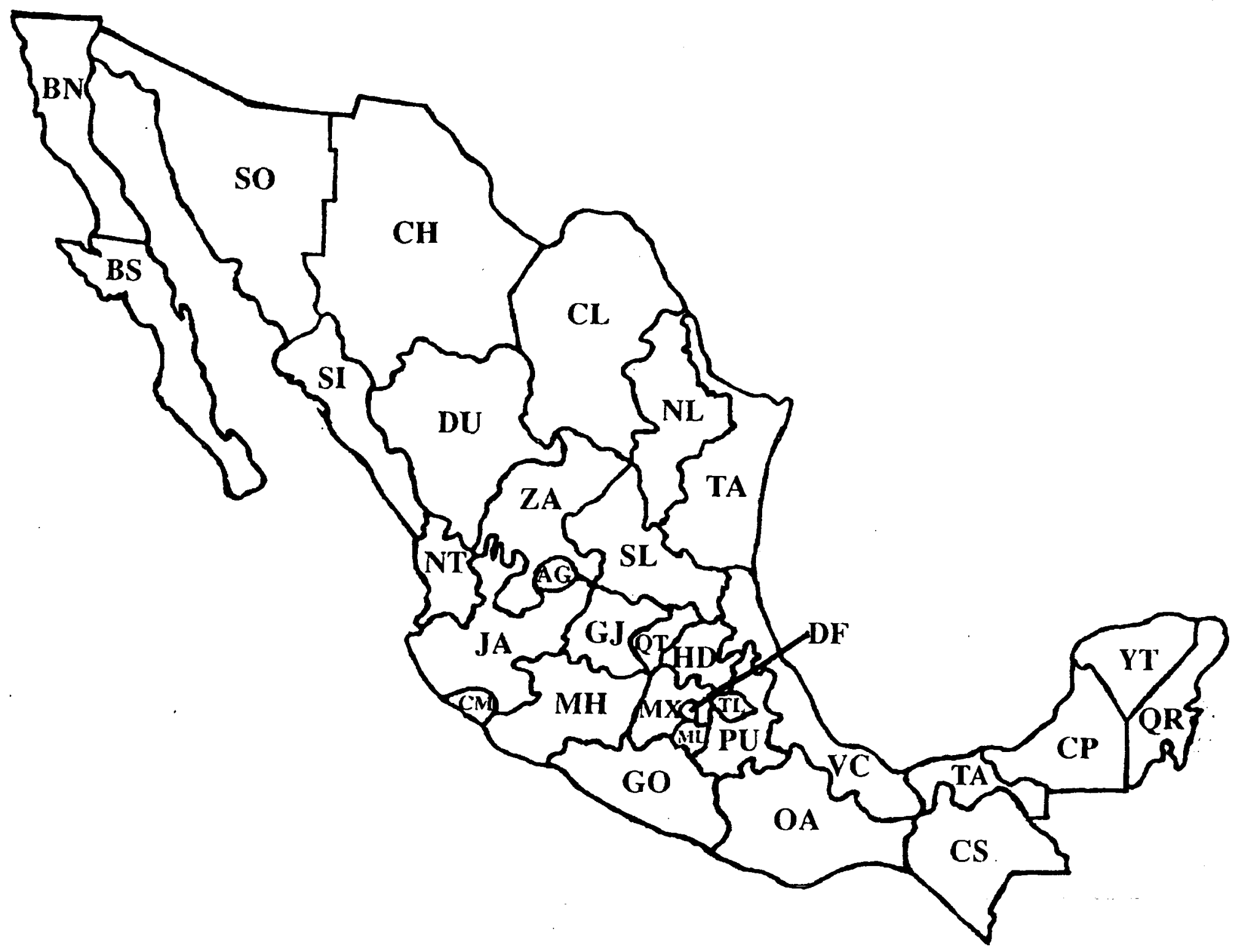

Fig. 1. State map of Mexico. State abbreviations are explained in Introduction.

Fig. 1. Carte politique du Mexique. L'abréviation des nọs d'états est détaillée dans l'introduction.

2.2. Americabaetis intermedius (Lugo-Ortiz \& McCafferty), 1994

MX ; OA [Río Atoyac at Ayoquezco, elev. 4,600', temp. $72^{\circ} \mathrm{F}, \mathrm{X}-22-1968$, R. K. Allen (larva)] ; VC [3 km W Zaculpan, elev. 6,000', IV-14-1960, M. Raymond Lee (larva)].

\subsection{Americabaetis lugoi Waltz \& McCafferty, 1999}

CS [stream at Santa Isabel 12 mi above Arriaga on Hwy 190, elev. $2000^{\prime}$, temp. $73^{\circ} \mathrm{F}, \mathrm{X}-23-1968$, R. K. Allen (larvae)].

\subsection{Americabaetis pleturus (Lugo-Ortiz \& McCafferty), 1994}

NL ; SL [Río Axtla at Comoco, $20 \mathrm{mi} \mathrm{N}$ Tamazunchale, VII-9-1966, R. K. Allen (larvae)]; TA ; VC [Río Paso de Ovejas at Paso de Ovejas, elev. 200', temp. 76 F, XI-101968, R. K. Allen (larva) ; Río Piedras Negras at Piedras Ne- gras, near Poza Rica, elev. 600', temp. 67(F, XI-12-1968, R. K. Allen (larva)].

\subsection{Apobaetis indeprensus Day, 1955}

TA.

\subsection{Baetis adonis Traver, 1935} $\mathrm{BN} ; \mathrm{BS} ; \mathrm{CH}$.

\subsection{Baetis flavistriga McDunnough, 1921 $\mathrm{CH}$.}

\subsection{Baetis magnus McCafferty \& Waltz, 1986}

CH ; CS ; DF ; DU ; GO [Río Acapulco 7 mi S Taxco, elev. 4,500', temp. $62^{\circ} \mathrm{F}$, XI-16-1968, R. K. Allen (larvae)] ; JA [Río La Pasion at Tizapan El Alto, elev. 5,100', temp. $72^{\circ} \mathrm{F}$, X-16-1968, R. K. Allen] ; MH [stream 9 mi E Morelia on 
Hwy 15, VII-5-1966, R. K. Allen (larvae)] ; MO ; MX ; NL ; $\mathrm{OA}$; PU ; SL ; VC.

\subsection{Baetis notos Allen \& Murvosh, 1987}

$\mathrm{MX}$; VC.

\subsection{Baetis tricaudatus Dodds, 1923}

BN ; DF ; JA [stream Hacienda Guadalupe, elev. 4,500', temp. 70 ${ }^{\circ}$ F, X-17-1968, R. K. Allen (larvae)] ; ML [Río Amcuzac at Huajintlan on Hwy 95, elev. 3,200', temp. 64 ${ }^{\circ} \mathrm{F}$, XI14-1968, R. K. Allen (larvae) ; Río Cuautla at Cuautla, elev. 4,600', temp. $70^{\circ} \mathrm{F}, \mathrm{XI}-13$ 1968, R. K. Allen (larva)] ; MX; OA [Río Atoyac at Ayoquezco, elev. 4000', temp. $72^{\circ} \mathrm{F}$. X22-1968 (larvae)] ; VC.

\subsection{Baetodes adustus Cohen \& Allen, 1972}

VC (type locality).

2.12. Baetodes bibranchius McCafferty \& Provonsha, 1993

TA.

2.13. Baetodes caritus Cohen \& Allen, 1972

$\mathrm{CH}$; $\mathrm{GO}$; $\mathrm{ML}$; VC.

2.14. Baetodes deficiens Cohen \& Allen, 1972

GO ; JA ; ML ; SO ; VC.

2.15. Baetodes edmundsi Koss, 1972

SI ; SO.

2.16. *Baetodes fortinensis Mayo, 1972

VC (type locality).

2.17. Baetodes fuscipes Cohen \& Allen, 1972

ML ; SI ; SO ; VC (type locality).

2.18. Baetodes inermis Cohen \& Allen, 1972

GO ; JA ; ML ; OA ; PU [creek at Hwy 106; Pahuatlan, VIII-17-1994, B. Kondratieff (larva)] ; TA (type locality) ; VC.

2.19. *Baetodes longus Mayo, 1973

BS ; NL ; TA (type locality).

2.20. *Baetodes obesus Mayo, 1972

VC (type locality).

2.21. Baetodes pallidus Cohen \& Allen, 1972

CS ; JA.

2.22. *Baetodes pictus Cohen \& Allen, 1972

$\mathrm{CH}$; VC (type locality).

\subsection{Baetodes tritus Cohen \& Allen, 1972}

CS ; GO [km 15 Carretera Bejucos cerca de Altamirano, XI-22-1984 (larvae) ; km 145 carretera Iguala, near Altamirano, XI-23-1984, H. Brailovsky et al. (larvae)] ; JA ; ML ; TA ; NL ; VC (type locality).
2.24. Baetodes velmae Cohen \& Allen, 1978

NL [Galeana, Municipiode Galeana, elev. 7,250', VII-81938, H. Hoogstraal (larva)].

\subsection{Callibaetis californicus Banks, 1900}

BN [Río Santo Toma, 2 mi S Santo Tomas, VI-8-1978, RKA \& CMM (larva)] ; BS [Arroyo Agua Caliente, $3 \mathrm{mi} \mathrm{E}$ Ejido Agua Caliente off Hwy \#1, VI-4-1978, RKA \& CMM (larvae)] ; GO ; MX ; ML ; NL.

2.26. Callibaetis distinctus Lugo-Ortiz \& McCafferty, 1996

SO (type locality).

2.27. Callibaetis floridanus Banks, 1900

$\mathrm{AG}$; $\mathrm{CS}$; $\mathrm{CH}$; DU ; GO ; HD ; JA ; ML ; NL ; OA ; TA ; $\mathrm{TC} ; \mathrm{VC}$.

\subsection{Callibaetis montanus Eaton, 1885}

BS $99 \mathrm{mi}$ NW Cabo San Lucas, unnamed stream, VI-71997, W. D. Shepard (larvae) ; Arroyo Agua Caliente, $3 \mathrm{mi} \mathrm{E}$ Ejido Agua Caliente off Hwy \#1, VI-4-1978, RKA \& CMM (larvae)] ; DF.

\subsection{Callibaetis pictus (Eaton), 1884}

BN [Río Santo Toma, 2 mi S Santo Tomas, VI-8-1978, RKA \& CMM (larva) ; 11 mi E Mike's Sky Rancho, unnamed stream, VI-3-1997, W. D. Shepard (larvae)] ; BS ; CH ; $\mathrm{CS}$; DF ; DU ; GO ; HD ; MH ; MO ; MX ; NL ; OA ; PU ; SI ; SO ; TA ; VC ; ZA [10 km antes de Tresnillo, VIII-171977, J. Bueno (larvae)].

2.30. Callibaetis punctilusus McCafferty \& Provonsha, 1993

$\mathrm{CH}$; NL ; SO [7.4 mi E Alamoso, rock hole off Cuchajaqui R, VI-5-1971, L. T. Nielsen \& T. J. Zavortiak (adult)].

2.31. Callibaetis undatus (Pictet), 1843

DF.

2.32. *Camelobaetidius arriaga (Traver \& Edmunds), 1968

BS ; CH ; CS (type locality) ; DU ; GO.

2.33. Camelobaetidius mexicanus (Traver \& Edmunds), 1968

BS 33 mi W Agua Caliente, VI-22-1997, W. D. Shepard (larva)] ; CS ; GO ; JA ; ML ; NL ; OA ; SI ; SO ; TA (type locality); VC ; ZA.

2.34. Camelobaetidius musseri (Traver \& Edmunds), 1968

BS [ 3 mi W Agua Caliente, Río Agua Caliente, VI-221997, W. D. Shepard (larvae)] ; CS ; GO ; JA ; ML ; NL ; OA ; SL ; VC (type locality).

2.35. *Camelobaetidius similis Lugo-Ortiz \& McCafferty, 1995

GO (type locality) ; OA [Río Atoyac at Ayoquezco, elev. 4,600', temp. $72^{\circ} \mathrm{F}, \mathrm{X}-22-1968, \mathrm{R}$. K. Allen (larva)] ; SI [Río 
Evora Mocorito near Mocorito on Hwy 21, I-17-1983, Allen, Murvosh, and Van Vector (larvae)] ; SO [Río Sonora $2 \mathrm{mi} \mathrm{SE}$ Rte 21 between Uras and Mazocahui, I-14-1983, Allen, Murvosh, and Van Vector (larva)] ; TA.

2.36. *Camelobaetidius sinaloa (Allen \& Murvosh), 1987 SI (type locality).

2.37. Camelobaetidius variablis Wiersema, 1997 NL ; TA.

2.38. Camelobaetidius warreni (Traver \& Edmunds), 1968 BS ; CS ; CH ; GO ; OA ; SI [Río Baluarte at Rosarito, I18-1983, Allen, Murvosh, and Van Vector (larvae)] ; SO.

2.39. Camelobaetidius sp. 1 McCafferty \& Davis, 1992

$\mathrm{CH} ; \mathrm{CL}$.

2.40. Cloeodes excogitatus Waltz \& McCafferty, 1987

BS [ 3 mi W. Agua Caliente, Río Agua Caliente, VI-221997, W. D. Shepard (larvae)] ; CS [stream at Santa Isabel, $12 \mathrm{mi}$ above Arriaga on Hwy 190, elev. 2,000', temp. $73^{\circ} \mathrm{F}$, X-23-1968, R. K. Allen (larvae)] ; NL ; SI [Río Evora Mocorito near Mocorito on Hwy 21, I-17-1983, Allen, Murvosh, and Van Vector (larvae) ; Río Baluarte at Rosarito, I-181983, Allen, Murvosh, Van Vector (larvae)] ; TA ; VC [Río Paso de Ovejas at Paso de Ovejas, elev. 200', temp. 76 $\mathrm{F}$, XI10-1968, R. K. Allen].

2.41. Cloeodes macrolamellus Waltz \& McCafferty, 1987 $\mathrm{CH}$; DU.

\subsection{2. *Cloeodes peninsulus Waltz \& McCafferty, 1987}

BS (type locality).

2.43. *Cloeodes waltzi Wiersema \& Baumgardner, 1999

TA (type locality).

2.44. *Fallceon eatoni (Kimmins), 1934

SO (type locality).

2.45. Fallceon fortipalpus Lugo-Ortiz \& McCafferty, 1999

TA.

\subsection{Fallceon longifolius (Kluge), 1992}

HD.

\subsection{Fallceon quilleri (Dodds), 1923}

BN ; BS ; CH ; CS ; GO [Río Balsas between Iguala and Chilpancingo on Hwy 95, elev. 1,800', temp. $76^{\circ} \mathrm{F}$, XI-161968, R. K. Allen (larvae)] ; MH [Río Turundeo at Turundeo on Hwy 15 near Tuxpan, elev. 6,633', VII-4-1966, R. K. Allen (larvae)] ; ML [stream $7 \mathrm{mi} S$ Cuemauaca, elev. 4,300' temp. $62^{\circ} \mathrm{F}$, XI-14-1968, R. K. Allen (larvae)] ; NL [Río Sabinas at Villaldama, elev. 1,500' temp. $80^{\circ} \mathrm{F}$, VIII-4-1970, R. K. Allen (larvae) ; Río Salinas at Cienega de Flores, elev. 1,450 ', VII-4-1966, R. K. Allen (larvae) ; Sabinas at Sabinas Hidalgo, elev. 1,180' temp. $70^{\circ}$ F, XI-25-1968, R. K. Allen (larvae) ; Río Camacho at Linares, elev. 1,350', temp. $84^{\circ} \mathrm{F}$,
VIII-5-1970, R. K. Allen (larva)] ; OA [Oaxaca, elev. 5,500', VII-28-1966, R. K. Allen (larvae) ; stream $6 \mathrm{mi}$ N Oaxaco, elev. 5,100', temp. $70^{\circ} \mathrm{F}, \mathrm{X}-22-1968$, R. K. Allen (larvae) ; Río Atoyac at Ayoquezco, elev. 4,600', temp. $72^{\circ} \mathrm{F}, \mathrm{X}-22-68$, R. K. Allen (larvae) ; stream $10 \mathrm{mi}$ N Huajuapan de Leon, elev. 5,400', temp. $70^{\circ} \mathrm{F}, \mathrm{XI}-7-1968$, R. K. Allen (larvae)] ; PU ; SL ; SI ; SO ; TA ; VC ; ZA [Río Juchipila at Juchipila, elev. 4,500', temp. 80 F, X-18-1968, R. K. Allen (larvae)].

\subsection{Moribaetis macaferti Waltz, 1985}

CS ; GO [trib. Río Papagayo near Tierra Colorado, elev. 500', temp. 86² F, XI-16-1968, R. K. Allen (larva)] ; MX [Río Los Barbechos, Nanchitla N. P. VIII-23-1994, B. Kondratieff (adult) ; OA ; PU ; VC.

\subsection{Moribaetis salvini (Eaton), 1885}

VC.

2.50. *Paracloeodes lugoi Randolph \& McCafferty, 2000 ML (type locality).

\subsection{Paracloeodes minutus (Daggy), 1945}

BS.

2.52. Plauditus virilis (McDunnough), 1923

TA [Río Purificacion near Hidalgo, elev. 1400', temp. $74^{\circ} \mathrm{F}$, XI-25-1968, R. K. Allen (larvae)].

2.53. Varipes lasiobrachius Lugo-Ortiz \& McCafferty, 1998

CS [stream at Santa Isabel $12 \mathrm{mi}$ above Arriaga on Hwy 190, elev. 2,000' temp., $73^{\circ} \mathrm{F}, 23-\mathrm{X}-1968$, R. K. Allen (larva)].

\section{Caenidae}

\subsection{Caenis anceps Traver, 1935}

VC.

\subsection{Caenis bajaensis Allen \& Murvosh, 1983}

BN [Mike's Sky Rancho, unnamed stream, VI-3-1997, W. D. Shepard (larva)] ; BS (type locality) ; CS ; DU ; NT ; SO.

\subsection{Caenis latipennis Banks, 1907}

CH ; CS ; NL ; SL [Ruinas de Tamuin, V-19-1979, J. Bueno (adults)] ; TA.

\subsection{Caenis punctata McDunnough, 1931}

$\mathrm{NL}$.

\section{Ephemeridae}

4.1. Hexagenia albivitta (Walker), 1853

VC.

\subsection{Hexagenia bilineata (Say), 1824}

SL. 
4.3. Hexagenia limbata (Serville), 1829

JA ; NL ; SL ; TA.

4.4. Hexagenia mexicana Eaton, 1885

$\mathrm{OA}$; VC ; (type locality : country only)

\section{Ephemerellidae}

5.1. Drunella coloradensis (Dodds), 1923

BN [Mike's Sky Rancho, Río San Rafael, VI-3-1997, W.

D. Shepard (larvae)].

5.2. Drunella flavilinea (McDunnough), 1926 BN.

5.3. Ephemerella altana Allen, 1968

BN.

5.4. Serratella micheneri (Traver), 1934

BN ; BS.

\section{Heptageniidae}

6.1. Epeorus margarita Edmunds \& Allen, 1964

$\mathrm{BN} ; \mathrm{BS} ; \mathrm{CH}$.

6.2. Epeorus metlacensis Traver, 1964

$\mathrm{DU} ; \mathrm{HD} ; \mathrm{MH}$; $\mathrm{MO}$; OA ; VC.

6.3. Ironodes nitidus (Eaton), 1885

BN.

6.4. *Nixe bella (Allen \& Cohen), 1977

VC (type locality).

6.5. *Nixe salvini (Kimmins), 1934

SO (type locality).

6.6. Rhithrogena morrisoni (Banks), 1924

BN.

6.7. Rhithrogena notialis Allen \& Cohen, 1977

DF (type locality); $\mathrm{MH} ; \mathrm{OA}$; VC.

6.8. Stenonema mexicanum integrum (McDunnough), 1924

TA.

6.9. Stenonema mexicanum mexicanum (Ulmer), 1920

TB (type locality).

\section{Isonychiidae}

7.1. Isonychia intermedia (Eaton), 1885

$\mathrm{CH}$.

7.2. Isonychia sicca Walsh, 1862

$\mathrm{CH}$; NL ; SL ; SO ; TA ; VC.

\section{Leptohyphidae}

8.1. *Leptohyphes alleni Brusca, 1971

OA (type locality).

8.2. *Leptohyphes berneri Traver, 1958

VC (type locality).

8.3. Leptohyphes brunneus Allen \& Brusca, 1973 CS ; GO ; JA ; ML ; OA (type locality) ; SO.

8.4. Leptohyphes castaneus Allen, 1967 OA.

8.5. *Leptohyphes dicinctus Allen \& Brusca, 1973 GO (type locality).

8.6. Leptohyphes lestes Allen \& Brusca, 1973 GO (type locality).

8.7. Leptohyphes michaeli Allen, 1978 $\mathrm{NL}$.

8.8. Leptohyphes mirus Allen, 1967

$\mathrm{CH}$; SI ; SO.

8.9. Leptohyphes nigripunctus Traver, 1943 GO.

8.10. Leptohyphes packeri Allen, 1967 $\mathrm{CH}$; $\mathrm{ML}$; NL ; NT ; OA ; SI ; SL ; SO ; TA ; VC.

8.11. * Leptohyphes pilosus Allen \& Brusca, 1973 VC (type locality).

8.12. Leptohyphes sabinas Traver, 1958 NL (type locality); TA ; VC.

8.13. *Leptohyphes spiculatus Allen \& Brusca, 1973 ML (type locality).

8.14. *Leptohyphes tarsos Allen \& Murvosh, 1987 SO (type locality).

8.15. Leptohyphes vescus Allen, 1978 TA.

8.16. Leptohyphes zalope Traver, 1958

$\mathrm{CH}$; CS ; GO (type locality) ; NL ; OA ; SL ; SO ; TA ; TB ; VC.

8.17. *Tricorythodes angulatus Traver, 1959

State Uncertain [Río Santa Lucia ; type locality] ; State Uncertain [San Pedro, 12-1840-1, L. Berner (adult)] ; SO [Río Chico, above El Chico, I-18-1988, B. C. Kondratieff and R. W. Baumann (adult)].

8.18. *Tricorythodes comus Traver, 1959

GO (type locality). 
8.19. Tricorythodes condylus Allen, 1967

$\mathrm{CH}$; SO.

8.20. Tricorythodes dimorphus Allen, 1967

$\mathrm{CH}$.

8.21. Tricorythodes explicatus (Eaton), 1892

$\mathrm{BN}$; BS ; CH ; SI ; SO.

8.22. Tricorythodes fictus Traver, 1935

CH [Río Gavilan, Gavilan Ranch, VIII-25-1986, B. C. Kondratieff (adult) ; Chuchugate Arroyo, trib. to Rio Chuhulpa, VI-23-1997, B. C. Kondratieff and R. W. Baumann (adults)]

8.23. *Tricorythodes mulaiki Traver, 1959

GO (type locality).

8.24. *Tricorythodes notatus Allen \& Brusca, 1973

ML (type locality); OA.

8.25. Tricorythodes texanus Traver, 1935

TA [paratypes misidentified as T. edmundsi Allen, 1967 (Wiersema pers. comm.)]

8.26. *Tricorythodes ulmeri Allen \& Brusca, 1973

ML (type locality).

\section{Leptophlebiidae}

9.1. Choroterpes inornata Eaton, 1892

$\mathrm{BN}$; BS ; $\mathrm{CH} ; \mathrm{OA}$; $\mathrm{SI}$; $\mathrm{SO}$ (type locality).

9.2. *Choroterpes ungulus Lugo-Ortiz \& McCafferty, 1996

CS (type locality).

9.3. *Farrodes maya Dominguez, 1999

CS (type locality).

9.4. *Farrodes mexicanus Dominguez, 1999

VC (type locality).

9.5. Farrodes otiesa Lugo-Ortiz \& McCafferty, 1996

TA [Río Guayalejo, X-25-1985, J. Bueno (larvae)].

9.6. Farrodes texanus Davis, 1987

TA.

9.7. *Farrodes tulija Dominguez, Molineri \& Peters, 1996

CS (type locality).

9.8. Hydrosmilodon primanus (Eaton), 1892

TB ; VC (type locality).

9.9. Neochoroterpes oklahoma (Traver), 1934

$\mathrm{CH}$; CS ; DU ; NL ; SL ; TA ; VC ; ZA.
9.10. *Neochoroterpes orientalis Henry, 1993

PU ; QT (type locality).

9.11. Paraleptophlebia memorialis (Eaton), 1884

BN.

9.12. Thraulodes brunneus Koss, 1966

$\mathrm{BN}$; BS ; CH ; DU ; GO ; JA ; MH ; MX ; ML ; OA ; SI ; $\mathrm{SO} ; \mathrm{VC}$.

9.13. *Thraulodes eccentricus Lugo-Ortiz \& McCafferty, 1996

CS ; DF ; GO ; HD (type locality) ; ML ; NL ; OA ; VC ; ZA.

9.14. *Thraulodes ephippiatus Traver \& Edmunds, 1967 CS (type locality).

9.15. Thraulodes gonzalesi Traver \& Edmunds, 1967 $\mathrm{CH}$; NL ; SI ; SO ; TA.

9.16. Thraulodes hilaris (Eaton), 1892

TB ; (type locality : country only).

9.17. Thraulodes humeralis Navás, 1935 (type locality : country only).

9.18. *Thraulodes lunatus Traver \& Edmunds, 1967 HD ; NL ; TA (type locality) ; ZA.

9.19. Thraulodes mexicanus (Eaton), 1884 (type locality : country only).

9.20. Thraulodes packeri Traver \& Edmunds, 1967 CS.

9.21. Thraulodes spangleri Traver \& Edmunds, 1967

CS ; VC [Tiapacoyan, Río Tomata, V-25-1985, J. Nagro (adults)].

9.22. Thraulodes speciosus Traver, 1934

$\mathrm{CH} ; \mathrm{GO} ; \mathrm{SO}$.

9.23. *Thraulodes tenulineus Lugo-Ortiz \& McCafferty, 1996

$\mathrm{CH}$; CS ; ML (type locality) ; NT ; OA ; SI ; SL ; SO ; TA ; $\mathrm{VC}$.

9.24. Thraulodes zonalis Traver \& Edmunds, 1967

CS.

9.25. Traverella albertana (McDunnough), 1931

$\mathrm{CH}$; CS ; GO ; HD ; JA ; ML ; SI ; SO ; TB ; VC ; ZA.

9.26. Traverella presidiana (Traver), 1934

CS ; NL ; TA.

9.27. Traverella promifrons Lugo-Ortiz \& McCafferty, 1996

CS ; NL. 
9.28. *Traverella sallei (Navas), 1935

(type locality : country only).

\section{Oligoneuriidae}

\subsection{Homoeoneuria alleni Pescador \& Peters, 1980} $\mathrm{CH}$.

\subsection{Homoeoneuria salviniae Eaton, 1881}

CS.

\section{3. * Lachlania iops Allen \& Cohen, 1977}

CS (type locality); SL.

10.4. Lachlania saskatchewanensis Ide, 1941 GO.

\section{Polymitarcyidae}

\subsection{Campsurus cuspidatus Eaton, 1871}

SL ; VC.

\subsection{Campsurus decoloratus (Hagen), 1861}

TA.

\subsection{Euthyplocia hecuba Hagen, 1861}

$\mathrm{CH} ; \mathrm{VC}$.

\section{Siphlonuridae}

\subsection{Siphlonurus occidentalis (Eaton), 1885}

$$
\mathrm{CH} ; \mathrm{HD} \text {; SO. }
$$

\section{Acknowledgments}

We thank R. Bauman (Provo, Utah), G. Edmunds (Salt Lake City, Utah), B. Kondratieff (Fort Collins, Colorado), W. Reeves (Clemson, South Carolina), and W. Shepard (Sacramento, California) for the donations that were used in this study. Also, we thank D. Baumgardner (College Station, Texas) for providing previously unpublished data. For aid in identification of certain specimens we thank C. Lugo-Ortiz (Grahamstown, South Africa) and N. Wiersema (Houston, Texas). This research was funded in part by the NSF Grant DEB-9901577. The paper has been assigned Purdue Agricultural Research Journal Number 16216

\section{References}

Allen R.K. 1967. - New species of New World Leptohyphinae (Ephemeroptera : Tricorythidae). Can. Entomol., 99 : 350-375.

Allen R.K. 1977. - A new species of Tricorythodes with notes (Ephemeroptera : Tricorythidae). J. Kans. entomol. Soc., 50 : 431-445.

Allen R.K. 1978. - The nymphs of North and Central American Leptohyphes (Ephemeroptera : Tricorythidae). Ann. entomol. Soc. Am., 71 : 537-558.

Allen R.K. 1985. - Mexican mayflies : new species, descriptions and records (Ephemeroptera). Pan-Pac. Entomol., 61 : 332-333.

Allen R.K. \& Brusca S.D. 1978. - Generic revisions of mayfly nymphs. II. Thraulodes in North and Central America (Leptophlebiidae). Can. Entomol., $110: 413-433$.
Allen R.K. \& Edmunds G.F. 1962. - A revision of the genus Ephemerella (Ephemeroptera, Ephemerellidae). V. The subgenus Drunella in North America. Misc. Publ. entomol. Soc. Am., 3 : 147-179.

Allen R.K. \& Cohen S.D. 1977. - Mayflies (Ephemeroptera) of Mexico and Central America : new species, descriptions, and records. Can. Entomol., $109: 399-414$.

Allen R.K. \& Murvosh C.M. 1983. - Taxonomy and zoogeography of the mayflies (Ephemeroptera : Insecta) of Baja California: Ann. entomol. Soc. Am., $76:$ 425-433.

Allen R.K. \& Murvosh C.M. 1987a. - Leptophlebiidae of the southwestern United States and northwestern Mexico (Insecta : Ephemeroptera). Great Basin Nat., 47 : 283-286.

Allen R.K. \& Murvosh C.M. 1987b. - New Baetidae from the southwestern United States and northern Mexico (Ephemeroptera : Insecta), with notes. Can. Entomol., 119 : 1095-1099.

Allen R.K. \& Murvosh C.M. 1987c. - Mayflies (Ephemeroptera : Tricorythidae) of the southwestern United States and northern Mexico. Ann. entomol. Soc. Amer., $80: 35-40$.

Anderson R.S. \& O'Brien C.W. 1996. - Curculionidae (Coleoptera). Pp. 329-349. In : J. Llorente Bousquets, A. N. García Aldrete E. \& González Soriano (eds.), Biodiversidad, taxonomía y biogeografía de artópodos de México : hacia una sintesis de su conociemento. Universidad Nacional Autonoma de Mexico, Mexico, D. F.

Baumgardner D.E. \& McCafferty W.P. 2000. - Leptohyphes zalope (Ephemeroptera : Leptohyphidae) : a polytypic North and Central American species. Entomol. News, $111: 49-59$.

Cohen S.D. \& Allen R.K. 1978. - Generic revisions of mayfly nymphs. III. Baetodes in North and Central America (Baetidae). J. Kans. entomol. Soc., 51 : 253-269.

Domínguez E. 1999. - Systematics, cladistics and biogeography of the American genus Farrodes (Ephemeroptera : Leptophlebiidae : Atalophlebiinae). Zool. J. Linn. Soc., 126 : 155-189.

Domínguez E., Molineri C. \& Peters W.L. 1996. - Ephemeroptera from Central and South America : new species of the Farrodes bimaculatus group with a key for the males. Stud. Neotrop. Fauna Environ., $31: 87-101$

Eaton A.E. 1881. - An announcement of new genera of the Ephemeridae. Entomol. monthly Mag., 17 : 191-197.

Eaton A.E. 1883-1888. - A revisional monograph of recent Ephemeridae or mayflies. Trans. Linn. Soc. Lond., 2nd Ser. Zool., 3 : $1-352$.

Edmunds G.F., Jr., Jensen S.L. \& Berner L. - 1976. The mayflies of North and Central America. Univ. Minn. Press, Minneapolis.

Lugo-Ortiz C.R. \& McCafferty W.P. 1994a. - New records of Ephemeroptera from Mexico. Entomol. News, 105 : 17-26.

Lugo-Ortiz C.R. \& McCafferty W.P. 1994b. - The mayfly genus Acerpenna (Insecta, Ephemeroptera, Baetidae) in Latin America. Stud. Neotrop. Fauna Environ., 29 : 65-74.

Lugo-Ortiz C.R. \& McCafferty W.P. 1995a. - New species, stage description, and records of Baetodes (Ephemeroptera : Baetidae) from Mexico and Central America. Entomol. News, 106 : 81-86.

Lugo-Ortiz C.R. \& McCafferty W.P. 1995b. - Taxonomy of the North and Central American species of Camelobaetidius (Ephemeroptera : Baetidae). Entomol. News, 106 : 178-192.

Lugo-Ortiz C.R. \& McCafferty W.P. 1996a. - New Central American and Mexican records of Ephemeroptera species. Entomol. News, 107 : 303-310.

Lugo-Ortiz C.R. \& McCafferty W.P. 1996b. - New species of Leptophlebiidae (Ephemeroptera) from Mexico and Central America. Annls. Limnol., 32 : 3-18.

Lugo-Ortiz C.R. \& McCafferty W.P. 1996c. - Contribution to the taxonomy of Callibaetis (Ephemeroptera : Baetidae) in southwestern North America and Middle America. Aquat. Insects, 18 : 1-9. 
Lugo-Ortiz C.R. \& McCafferty W.P. 1998. - Five new genera of Baetidae (Insecta : Ephemeroptera) from South America. Annls Limnol., 34 : 57-73.

Lugo-Ortiz C.R., McCafferty W.P. \& Waltz R.D. 1994. - Contribution to the taxonomy of the Panamerican genus Fallceon (Ephemeroptera : Baetidae). J. N. Y. entomol. Soc., $102: 460-475$.

McCafferty W.P. 1985. — New records of Ephemeroptera from Middle America. Internat. Quat. Entomol., 1 : 9-11.

McCafferty W.P. 1996. - The Ephemeroptera species of North America and index to their complete nomenclature. Trans. Am. entomol. Soc., 122 : 1-54.

McCafferty W.P. 1998. - Ephemeroptera and the great American interchange. J. N. Am. benthol. Soc., $17: 1-20$.

McCafferty W.P. \& Lugo-Ortiz C.R. 1996. - Ephemeroptera. Pp. 133-145. In : J. Llorente Bousquets, A.N. García Aldrete \& E. González Soriano (eds.), Biodiversidad, taxonomía y biogeografía de artrópodos de México : hacia una sintesis de su conocimiento. Universidad Nacional Autonoma de Mexico, Mexico D.F.

McCafferty. W.P. \& Lugo-Ortiz C.R. 1998. - The adult of Moribaetis macaferti (Ephemeroptera : Baetidae). Entomol. News, $109: 117-121$

McCafferty W.P. \& Randolph R.P. 1998. — Canada mayflies : a faunistic compendium. Proc. entomol. Soc. Ontario, 129 : 1-51.

McCafferty W.P. \& Randolph R.P. 2000. - Further contributions to the spatulate clawed Baetidae (Ephemeroptera). Entomol. News, in press.

McCafferty W.P., Flowers R.W. \& Waltz R.D. 1992. — The biogeography of Mesoamerican mayflies. Pp. 173-193. In : S.P. Darwin \& A.L. Weldon (eds.), Biogeography of Mesoamerica : proceedings of a symposium. Tulane Univ. Stud. Zool. Bot., Suppl. Publ. 1.

McCafferty W.P., Lugo-Ortiz C.R., Provonsha A.V. \& Wang T-Q. 1997. - Los Efemeropteros de Mexico : I. Classificacion superior, diagnosis de familias y composicion. Dugesiana, 4 : 1-29.

McCafferty W.P., Lugo-Ortiz C.R. \& Jacobi G.Z. 1997. - Mayfly fauna of New Mexico. Great Basin Natural., $57: 283-314$.

Mittermeier R.A. 1988. - Primate diversity and the tropical forest : case studies from Brazil and Madagascar and the importance of the megadiversity countries. Pp. 145-154. In : E. O. Wilson (ed.), Biodiversity. National Academic Press, Washington, DC.

Navás L. 1935. - Efemeropteros. Decadas de Insectos Nuevos. Broteria Ciencias Naurais, 99-103.

Pescador M.L. \& Peters W.L. 1980 - A revision of the genus Homoeoneuria (Ephemeroptera : Oligoneuriidae). Trans. Am. entomol. Soc., 106 : 357-393.

Peters W.L. \& Edmunds G.F., Jr. 1970. - Revision of the generic classification of the Eastern Hemisphere Leptophlebiidae (Ephemeroptera). Pac. Insects, $12:$ 157-240.

Ramamoorthy T.P., Bye R., Lot A. \& Fa J. 1993. - Introduction. Pp. xix-xxxix. In : T. P. Ramamoorthy, R. Bye, A. Lot \& F. Fa (eds.), Biological diversity of Mexico, origins and distributions. Oxford Univ. Press, New York.

Randolph R.P. \& McCafferty W.P. 1998. - Diversity and distribution of the mayflies (Ephemeroptera) of Illinois, Indiana, Kentucky, Michigan, Ohio, and Wisconsin. Ohio Biol. Surv. Bull. New Ser., 13: 1-188.

Randolph R.P. \& McCafferty W.P. 2000. - A New Species of Paracloeodes (Ephemeroptera : Baetidae) from Mexico. Entomol. News, 111 : 133-136.

Traver J.R. 1935. — Part II, Systematics. Pp. 237-739. In : J.G. Needham, J.R. Traver \& Y.-C. Hsu (eds.), The biology of mayflies. Comstock Publ. Co., Ithaca, New York.

Waltz R.D. \& McCafferty W.P. 1987. - Revision of the genus Cloeodes Traver (Ephemeroptera : Baetidae). Ann. entomol. Soc. Am., 80 : 191-207.

Waltz R.D. \& McCafferty W.P. 1999. - Additions to the taxonomy of Americabaetis (Ephemeroptera : Baetidae) : A. lugoi, n. sp., adult of $A$. robacki, and key to larvae. Entomol. News, 110 : 39-44.

Wiersema N. 1998. - Camelobaetidius variabilis (Ephemeroptera : Baetidae) : a new species from Texas, Oklahoma, and Mexico. Entomol. News, $109: 21-26$.

Wiersema N.A. \& Baumgardner D.E. 2000. - Distributional and taxonomical contributions to the Ephemeroptera fauna of Mexico and Central America. Entomol. News, 111 : 60-66. 